\title{
Metacognitive Awareness Skill Level of Athletes and Sedentary Turkish University Students in Both Genders
}

Afyon Kocatepe University, School of Physical Education and Sports, Afyonkarahisar, Turkey. Email:sgolunuk@gmail.com

${ }^{2}$ Mersin University, Faculty of Sports Sciences, Mersin, Turkey.

Email:mziyagil@gmail.com

\section{Abstract}

Due to limited numbers of published studies regarding the relationship between sports and cognitive functions in adolescents. So, this study compares the metacognitive awareness skill (MAS) levels between athletes and sedentary Turkish University Students. Data were collected from 434 voluntary male and 493 female university students by MAS inventory including sub-dimensions of knowledge about cognition and the regulation of cognition. Mann Whitney $U$ tests were used for comparison between two groups. Also, Spearman rank order correlations were performed to analyze the relations between participating sports activities and sub-dimensions of MAS. This study showed that there was no significant difference in the favor of athlete participants in all sub-dimensions in male and female group. In females, sedentary participants had significantly higher values than athlete counterparts in all sub-dimensions of MAS. In sedentary group, females had significantly higher values than males in the sub-dimensions of declarative knowledge, procedural knowledge, conditional knowledge and evaluation while males had significantly higher values than females in all sub-dimensions except debugging strategies in athlete group. Also, there were negative significant relationships between participation sport activities and all sub-dimensions of MASI scores in females while observing no significant relations among variables in males.: It can be concluded that females in the sedentary group and male in the athlete group had significantly higher MAS values. Participation in sport activities had no positive effect on MAS scores in both genders.

Keywords: Metacognitive awareness skills, Participation sport, Gender, Knowledge about cognition, The regulation of cognition.

Citation | Sebiha Gölünük Başpinar; Mehmet Akif Ziyagil (2019). Metacognitive Awareness Skill Level of Athletes and Sedentary Turkish University Students in Both Genders. Asian Journal of Education and Training, 5(4): 555-561

History:

Received: 5 August 2019

Revised: 9 September 2019

Accepted: 11 October 2019

Published: 25 November 2019

Licensed: This work is licensed under a Creative Commons

Attribution 3.0 License (oc) EY

Publisher: Asian Online Journal Publishing Group
Acknowledgement: Both authors contributed to the conception and design of the study.

Funding: This study received no specific financial support.

Competing Interests: The authors declare that they have no conflict of interests.

Transparency: The authors confirm that the manuscript is an honest, accurate, and transparent account of the study was reported; that no vital features of the study have been omitted; and that any discrepancies from the study as planned have been explained.

Ethical: This study follows all ethical practices during writing.

\section{Contents}

1. Introduction

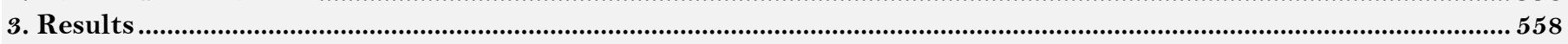

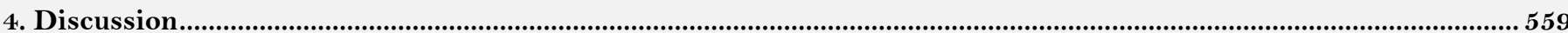

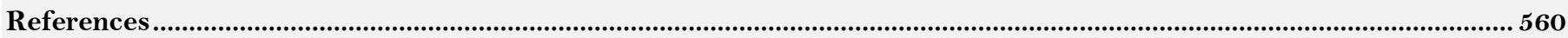




\section{Contribution of this paper to the literature}

This study contributes to the existing literature by investigating gender and sportive activity related differences to the metacognitive awareness skill level in both athletes and sedentary groups.

\section{Introduction}

The ability of the individual to control, regulate and guide the cognitive processes at the highest level is defined as metacognition (Flavell, 1979). Executions of efficient technical and tactical skills in sportive activities depend partly on consciously arranging decision-making process as part of perceptual-cognitive function. Integration of technical and tactical skills into related sport context needs intellectual processes including perceptual motor skills, knowledge and decision making. These components constitute successful sport performance (Mitchell et al., 1994). Erickson et al. (2019) stated that physical activity improves cognitive and brain functions, but there are different views on the consistency and magnitude of the effect. They concluded that there are supportive views ranging from moderate to strong that physical activity benefits cognitive functioning in early and late life and in some populations characterized by cognitive deficits Erickson et al. (2019).

Two components constitute metacognitive awareness skills inventory. First component of MAS inventory is the knowledge about cognition with including sub-dimensions of declarative knowledge, procedural knowledge and conditional knowledge. Second component of MAS inventory is the regulation of cognition with including subdimensions of planning, information management strategies, comprehension monitoring, debugging strategies and evaluation (Schraw and Moshman, 1995). In most individual and team sports, metacognitive awareness skills make possible the optimal performance related to open or closed skills with the integration of technical and tactical skills into match and game context.

Research findings on the relationship between lack of physical activity and mental health variables are increasing. It has been reported that overweight sedentary individuals are more prone to risky behaviors such as the habit of using alcohol and drugs and suicide attempts (Kubesch, 2002; Lipowski et al., 2016).

Cognitive functions include memory, attention, visual-spatial and as cognitive control and supervisory attentional system, while complex cognitive processes relate to abstraction, cause-effect, creative thinking and thinking functions, including planning and speech. Although the relationship between regular participation in sporting activities and cognitive functioning is known (Ellemberg and St-Louis-Deschênes, 2010) partially incorrect designed studies have reported conflicting results on the effect of sport on cognitive functions. Some of these studies report positive effects, while other studies do not confirm the positive effect of sportive activity on cognitive functions (Ahamed et al., 2007; Fels et al., 2015; Lambrick et al., 2016).

In other side, studies showed inconsistent results regarding differences in metacognitive skills levels between male and female students. Some studies have reported that metacognitive skills of boys and girls differ, while other studies do not show significant differences (Niemivirta, 1997; Bidjerano, 2005; Virtanen and Nevgi, 2010). These findings demonstrate that both girls and boys use their metacognitive skills during learning (Niemivirta, 1997; Bidjerano, 2005; Virtanen and Nevgi, 2010).

The findings of the research conducted by Liliana and Lavinia (2011) demonstrated that both girls and boys used metacognitive skills during learning. In this study, as a result of the motivation and effort of a student there were significant differences between boys and girls in the sub-dimensions of performance perception, perceptions of teachers about learning expectations, use of previous knowledge in problems, solving their own intellectual strengths and weaknesses, planning, accumulation of knowledge, using various learning strategies and monitoring of learning process. Such information may be useful in developing educational programs for young university students to develop their cognitive functions that are important to a particular sport discipline. Thus, it seems possible that these results of studies can be used in educational applications including teaching and learning in sport context. There are limited number of studies in the literature showing that there are differences in metacognitive skills between male and female university students in the integrity of gender and participation in sports activities. Our study aims to investigate the potential gender and sport participation related differences in metacognitive awareness skills of male and female university students.

Thus, this study compares the metacognitive awareness skill levels between athletes and sedentary in Turkish University male and female students.

\section{Material and Method}

This descriptive study aims to compare the metacognitive skills levels between athletes and sedentary male and female university students in autumn semester of 2018-20198 at Afyon Kocatepe University.

\subsection{Participants}

Data were collected from 434 voluntary male and 493 female university students. Male participants consisted of 347 sedentary and 87 athletes while female participants were composed of 290 sedentary and 203 athletes. The athlete participants were students from coaching and recreation departments of the Faculty of Sport Sciences, while the sedentary participants were students from the faculty of education and the faculty of economics and administrative sciences. The study was approved by the ethics committee of the Afyon Kocatepe University.

\subsection{Data Collection Instrument}

Metacognitive Awareness Skills (MAS) inventory was used for comparison of the metacognitive awareness skill levels between sedentary and athletes university students in male and females. This inventory was developed by Schraw and Dennison (1994) and translated into Turkish by Akın et al. (2007). MASI is self-report inventory and consisted of two components. First component is the knowledge about cognition while second is the regulation of cognition. First component of MAS contains 17 questions and three sub-dimensions including declarative knowledge, procedural knowledge and conditional knowledge. In Second component of MASI, there are 
35 questions and 5 sub-dimensions including planning, information management strategies, comprehension monitoring, debugging strategies and evaluation. The lowest score is 52 points while highest score is 260 points in MASI with 5 points Likert type scale. This instrument ranges from 1 (always false) to 5 (always true). Low scores demonstrate weak metacognitive awareness while high scores indicate strong metacognitive awareness. There is no reverse coding in MASI. In the reliability study of the inventory, the Cronbach alpha coefficient and test retest reliability were 0.95 and, 0.95 , respectively (Akın et al., 2007).

\begin{tabular}{|c|c|c|c|c|c|c|c|c|c|c|}
\hline $\begin{array}{l}\text { Sub- } \\
\text { dimensions }\end{array}$ & Variables & Groups & $\mathbf{N}$ & $\mathbf{M}$ & SD & Min. & Max. & MWU & z-value & Sig \\
\hline & Age (years) & Sedentary & 347 & 20,86 & 2,80 & 17,00 & 42,00 & & & \\
\hline & & Athlete & 87 & 21,38 & 2,84 & 18,00 & 29,00 & 13496,0 & $-1,551$ &, 121 \\
\hline & & Total & 434 & 20,97 & 2,82 & $17, \mathrm{OO}$ & 42,00 & & & \\
\hline \multirow{9}{*}{$\begin{array}{l}\text { Knowledge } \\
\text { about } \\
\text { cognition }\end{array}$} & \multirow{3}{*}{$\begin{array}{l}\text { Declarative Knowledge } \\
\text { (8-40 points) }\end{array}$} & Sedentary & 347 & 26,74 & $6,4+1$ & 9,00 & 40,00 & \multirow{3}{*}{14173,5} & \multirow{3}{*}{,- 882} & \multirow{3}{*}{,378 } \\
\hline & & Athlete & 87 & 27,41 & 5,78 & 14,00 & 38,00 & & & \\
\hline & & Total & 434 & 26,87 & 6,29 & 9,00 & 40,00 & & & \\
\hline & \multirow{3}{*}{$\begin{array}{l}\text { Procedural Knowledge } \\
(4-20 \text { points })\end{array}$} & Sedentary & 347 & 13,39 & 3,32 & 4,00 & 20,00 & \multirow{3}{*}{14194,0} & \multirow{3}{*}{,- 865} & \multirow{3}{*}{,387 } \\
\hline & & Athlete & 87 & 13,71 & 3,64 & 6,00 & 20,00 & & & \\
\hline & & Total & 434 & 13,45 & 3,38 & 4,00 & 20,00 & & & \\
\hline & \multirow{3}{*}{$\begin{array}{l}\text { Conditional Knowledge } \\
\text { (5-25 points) }\end{array}$} & Sedentary & 347 & 16,62 & 4,03 & 7,00 & 25,00 & \multirow{3}{*}{14722,5} & \multirow{3}{*}{,- 357} & \multirow{3}{*}{, 721} \\
\hline & & Athlete & 87 & 16,75 & 3,81 & 4,00 & 25,00 & & & \\
\hline & & Total & 434 & 16,65 & 3,98 & 4,00 & 25,00 & & & \\
\hline \multirow{15}{*}{$\begin{array}{l}\text { Regulation of } \\
\text { cognition }\end{array}$} & \multirow{3}{*}{$\begin{array}{l}\text { Planning } \\
\text { (7-35 points) }\end{array}$} & Sedentary & 347 & 23,46 & 5,37 & 9,00 & 35,00 & \multirow{3}{*}{15048,0} & \multirow{3}{*}{,- 045} & \multirow{3}{*}{,964 } \\
\hline & & Athlete & 87 & 23,48 & 5,00 & 8,00 & 33,00 & & & \\
\hline & & Total & 434 & 23,46 & 5,29 & 8,00 & 35,00 & & & \\
\hline & \multirow{3}{*}{$\begin{array}{l}\text { Information Management } \\
\text { Strategies } \\
\text { (9-45 points) }\end{array}$} & Sedentary & 347 & 31,63 & 5,80 & 16,00 & 65,00 & \multirow{3}{*}{14523,0} & \multirow{3}{*}{,- 547} & \multirow{3}{*}{, 584} \\
\hline & & Athlete & 87 & 31,72 & 5,35 & 15,00 & 45,00 & & & \\
\hline & & Total & 434 & 31,65 & 5,71 & 15,00 & 65,00 & & & \\
\hline & \multirow{3}{*}{\begin{tabular}{|l|} 
Comprehension \\
Monitoring \\
(8-40 points) \\
\end{tabular}} & Sedentary & 347 & 26,99 & 5,35 & 14,00 & 40,00 & \multirow{3}{*}{14952,0} & \multirow{3}{*}{,- 136} & \multirow{3}{*}{,891 } \\
\hline & & Athlete & 87 & 27,06 & 5,22 & 15,00 & 38,00 & & & \\
\hline & & Total & 434 & 27,01 & 5,31 & $14, \mathrm{OO}$ & 40,00 & & & \\
\hline & \multirow{3}{*}{$\begin{array}{l}\text { Debugging Strategies } \\
\text { (5-25 points) }\end{array}$} & Sedentary & 347 & 17,94 & 3,55 & 7,00 & 25,00 & \multirow{3}{*}{14845,5} & \multirow{3}{*}{,- 239} & \multirow{3}{*}{, 811} \\
\hline & & Athlete & 87 & 17,78 & 3,68 & 5,00 & 25,00 & & & \\
\hline & & Total & 434 & 17,91 & 3,57 & 5,00 & 25,00 & & & \\
\hline & \begin{tabular}{|l|} 
Evaluation \\
\end{tabular} & Sedentary & 347 & 20,55 & 4,15 & 8,00 & 30,00 & & & \\
\hline & (6-30 points) & Athlete & 87 & 20,55 & 3,77 & 12,00 & 30,00 & 14959,0 &,- 130 & ,897 \\
\hline & & Total & 434 & 20,55 & 4,07 & 8,00 & 30,00 & & & \\
\hline
\end{tabular}

**There was significant difference between two groups at $\mathrm{P}<0.01$ level.

\begin{tabular}{|c|c|c|c|c|c|c|c|c|c|c|}
\hline $\begin{array}{l}\text { Sub- } \\
\text { dimensions }\end{array}$ & Variables & Groups & $\mathbf{N}$ & $\mathbf{M}$ & SD & Min. & Max. & MWU & z-value & Sig \\
\hline & \multirow[t]{3}{*}{ Age (years) } & Sedentary & 290 & 20,91 & 2,12 & 17,00 & 33,00 & \multirow{3}{*}{29357,0} & \multirow{3}{*}{,- 051} & \multirow{3}{*}{,959 } \\
\hline & & Athlete & 203 & 20,81 & 1,76 & 17,00 & 26,00 & & & \\
\hline & & Total & 493 & 20,87 & 1,97 & 17,00 & 33,00 & & & \\
\hline \multirow{9}{*}{$\begin{array}{l}\text { Knowledge } \\
\text { about } \\
\text { cognition }\end{array}$} & \multirow{3}{*}{$\begin{array}{l}\text { Declarative Knowledge } \\
\text { (8-40 points) }\end{array}$} & Sedentary & 290 & 28,15 & 6,21 & $11, \mathrm{OO}$ & 51,00 & \multirow{3}{*}{20851,5} & \multirow{3}{*}{$-5,521$} & \multirow{3}{*}{, $000 * *$} \\
\hline & & Athlete & 203 & 24,66 & 6,99 & 10,00 & 40,00 & & & \\
\hline & & Total & 493 & 26,71 & 6,76 & 10,00 & 51,00 & & & \\
\hline & \multirow{3}{*}{$\begin{array}{l}\text { Procedural Knowledge } \\
\text { (4-20 points) }\end{array}$} & Sedentary & 290 & 13,96 & 3,23 & $5, \mathrm{OO}$ & 20,00 & \multirow{3}{*}{22760,5} & \multirow{3}{*}{$-4,306$} & \multirow{3}{*}{, $000 * *$} \\
\hline & & Athlete & 203 & 12,50 & 3,60 & 5,00 & 20,00 & & & \\
\hline & & Total & 493 & 13,36 & 3,46 & $5, \mathrm{OO}$ & 20,00 & & & \\
\hline & \multirow{3}{*}{$\begin{array}{l}\text { Conditional Knowledge } \\
\text { (5-25 points) }\end{array}$} & Sedentary & 290 & 17,58 & 3,84 & 4,00 & 28,00 & \multirow{3}{*}{21509,5} & \multirow{3}{*}{$-5,109$} & \multirow{3}{*}{,OOO** } \\
\hline & & Athlete & 203 & 15,50 & 4,36 & 6,00 & 25,00 & & & \\
\hline & & Total & 493 & 16,72 & 4,19 & 4,00 & 28,00 & & & \\
\hline \multirow{15}{*}{$\begin{array}{l}\text { Regulation of } \\
\text { cognition }\end{array}$} & \multirow{3}{*}{$\begin{array}{l}\text { Planning } \\
\text { (7-35 points) }\end{array}$} & Sedentary & 290 & 23,96 & 5,04 & 8,00 & 35,00 & \multirow{3}{*}{22287,5} & \multirow{3}{*}{$-4,600$} & \multirow{3}{*}{, $\mathrm{OOO}^{* *}$} \\
\hline & & Athlete & 203 & 21,52 & 6,07 & $7, \mathrm{OO}$ & 35,00 & & & \\
\hline & & Total & 493 & 22,96 & 5,61 & 7,00 & 35,00 & & & \\
\hline & Information Management & t Sedentary & 290 & 32,01 & 5,73 & 14,00 & 45,00 & \multirow{3}{*}{24391,0} & \multirow{3}{*}{$-3,245$} & \multirow{3}{*}{,OO $1 * *$} \\
\hline & \multirow{2}{*}{$\begin{array}{l}\text { Strategies } \\
(9-45 \text { points })\end{array}$} & Athlete & 203 & 30,32 & 6,64 & 14,00 & 63,00 & & & \\
\hline & & Total & 493 & 31,31 & 6,17 & 14,00 & 63,00 & & & \\
\hline & \multirow{3}{*}{$\begin{array}{l}\text { Comprehension } \\
\text { Monitoring } \\
(8-40 \text { points })\end{array}$} & Sedentary & 290 & 27,52 & 5,33 & 14,00 & 40,00 & \multirow{3}{*}{23458,0} & \multirow{3}{*}{$-3,845$} & \multirow{3}{*}{, $000 * *$} \\
\hline & & Athlete & 203 & 25,33 & 6,23 & 11,00 & 40,00 & & & \\
\hline & & Total & 493 & 26,62 & 5,81 & 11,00 & 40,00 & & & \\
\hline & Debugging Strategies & Sedentary & 290 & 18,16 & 3,33 & $5, \mathrm{OO}$ & 25,00 & & & \\
\hline & (5-25 points) & Athlete & 203 & 17,20 & 3,63 & 8,00 & 25,00 & 25105,0 & $-2,794$ &, $005 * *$ \\
\hline & & Total & 493 & 17,76 & 3,48 & 5,00 & 25,00 & & & \\
\hline & Evaluation & Sedentary & 290 & 21,21 & 4,04 & 10,00 & 30,00 & & & \\
\hline & (6-30 points) & Athlete & 203 & 19,20 & 4,46 & 9,00 & 30,00 & 21903,5 & $-4,852$ & ,OOO** \\
\hline & & Total & 493 & 20,38 & 4,33 & 9,00 & 30,00 & & & \\
\hline
\end{tabular}

**There was significant difference between two groups at $\mathrm{P}<0.01$ level. 


\subsection{Data Analysis}

In absence of normal data distribution, nonparametric Mann Whitney U tests were performed for comparisons between athlete and sedentary participants. In addition, Spearman rank order correlations were used to test relations between metacognitive skills level and sport participation in both genders.

\section{Results}

This study compares the metacognitive awareness skill levels between athletes and sedentary Turkish male and female university students. In this context, Comparison of metacognitive skill levels between athletes and sedentary participants in Turkish male university students. was demonstrated in Table 1. Also, Comparison of metacognitive skill levels between athletes and sedentary participants in Turkish female university students was shown in Table 2. In addition, comparison of metacognitive skill levels between males and females participants in sedentary group was presented in Table 3 while comparison of metacognitive skill levels between males and females participants in athlete group was demonstrated in Table 4. Also, Spearman rank order correlation coefficients among sport participation and metacognitive variables in Turkish male and female university students was presented in Table 5.

\begin{tabular}{|c|c|c|c|c|c|c|c|c|c|c|}
\hline $\begin{array}{l}\text { Sub- } \\
\text { dimensions }\end{array}$ & Variables & Groups & $\mathbf{N}$ & $\mathbf{M}$ & SD & Min. & Max. & MWU & z-value & Sig \\
\hline & \multirow{3}{*}{ Age (years) } & Males & 347 & 20,86 & 2,80 & 17,00 & 42,00 & \multirow{3}{*}{46597,0} & \multirow{3}{*}{$-1,631$} & \multirow{3}{*}{, 103} \\
\hline & & Females & 290 & 20,91 & 2,12 & 17,00 & 33,00 & & & \\
\hline & & Total & 637 & 20,88 & 2,51 & 17,00 & 42,00 & & & \\
\hline \multirow{9}{*}{$\begin{array}{l}\text { Knowledge } \\
\text { about } \\
\text { cognition }\end{array}$} & \multirow{3}{*}{$\begin{array}{l}\text { Declarative Knowledge } \\
\text { (8-40 points) }\end{array}$} & Males & 347 & 26,74 & 6,41 & 9,00 & 40,00 & \multirow{3}{*}{43907,5} & \multirow{3}{*}{$-2,774$} & \multirow{3}{*}{, $006^{* *}$} \\
\hline & & Females & 290 & 28,15 & 6,21 & $11, \mathrm{OO}$ & 51,00 & & & \\
\hline & & Total & 637 & 27,38 & 6,35 & 9,00 & 51,00 & & & \\
\hline & \multirow{3}{*}{$\begin{array}{l}\text { Procedural Knowledge } \\
\text { (4-20 points) }\end{array}$} & Males & 347 & 13,39 & 3,32 & 4,00 & 20,00 & \multirow{3}{*}{45540,0} & \multirow{3}{*}{$-2,073$} & \multirow{3}{*}{,038* } \\
\hline & & Females & 290 & 13,96 & 3,23 & 5,00 & 20,00 & & & \\
\hline & & Total & 637 & 13,65 & 3,29 & 4,00 & 20,00 & & & \\
\hline & \multirow{3}{*}{$\begin{array}{l}\text { Conditional Knowledge } \\
\text { (5-25 points) }\end{array}$} & Males & 347 & 16,62 & 4,03 & 7,00 & 25,00 & \multirow{3}{*}{43549,5} & \multirow{3}{*}{$-2,935$} & \multirow{3}{*}{,003 $* *$} \\
\hline & & Females & 290 & 17,58 & 3,84 & 4,00 & 28,00 & & & \\
\hline & & Total & 637 & 17,06 & 3,97 & 4,00 & 28,00 & & & \\
\hline \multirow{15}{*}{$\begin{array}{l}\text { Regulation of } \\
\text { cognition }\end{array}$} & \multirow{3}{*}{$\begin{array}{l}\text { Planning } \\
\text { (7-35 points) }\end{array}$} & Males & 347 & 23,46 & 5,37 & 9,00 & 35,00 & \multirow{3}{*}{47628,5} & \multirow{3}{*}{$-1,163$} & \multirow{3}{*}{, 245} \\
\hline & & Females & 290 & 23,96 & 5,04 & 8,00 & 35,00 & & & \\
\hline & & Total & 637 & 23,69 & 5,22 & 8,00 & 35,00 & & & \\
\hline & Information & Males & 347 & 31,63 & 5,80 & 16,00 & 65,00 & & & \\
\hline & Management Strategies & Females & 290 & 32,01 & 5,73 & $14, \mathrm{OO}$ & 45,00 & 48154,5 &,- 936 & ,349 \\
\hline & (9-45 points) & Total & 637 & 31,80 & 5,77 & $14, \mathrm{OO}$ & 65,00 & & & \\
\hline & Comprehension & Males & 347 & 26,99 & 5,35 & $14, \mathrm{OO}$ & 40,00 & & & \\
\hline & Monitoring & Females & 290 & 27,52 & 5,33 & 14,00 & 40,00 & 47558,5 & $-1,194$ & ,233 \\
\hline & (8-40 points) & Total & 637 & 27,23 & 5,34 & 14,00 & 40,00 & & & \\
\hline & & Males & 347 & 17,94 & 3,55 & 7,00 & 25,00 & & & \\
\hline & Debugging strategies & Females & 290 & 18,16 & 3,33 & 5,00 & 25,00 & 48877,5 &,- 624 &, 533 \\
\hline & & Total & 637 & 18,04 & 3,45 & 5,00 & 25,00 & & & \\
\hline & & Males & 347 & 20,55 & 4,15 & 8,00 & 30,00 & & & \\
\hline & Evaluation & Females & 290 & 21,21 & 4,04 & 10,00 & 30,00 & 45736,5 & $-1,985$ &, $047 *$ \\
\hline & & Total & 637 & 20,85 & 4,11 & 8,00 & 30,00 & & & \\
\hline
\end{tabular}

There was significant difference between two groups at $\mathrm{P}<0.05$ level.

Table-4. Comparison of metacognitive awareness skill levels between males and females participants in athlete group.

\begin{tabular}{|c|c|c|c|c|c|c|c|c|c|c|}
\hline$\overline{\text { Sub-dimensions }}$ & Variables & Groups & $\mathbf{N}$ & $\mathbf{M}$ & SD & Min. & Max. & MWU & z-value & Sig \\
\hline & \multirow{3}{*}{ Age (years) } & Males & 87 & 21,38 & 2,84 & 18,00 & 29,00 & \multirow{3}{*}{8446,5} & \multirow{3}{*}{,- 594} & \multirow{3}{*}{, 552} \\
\hline & & Females & 203 & 20,81 & 1,76 & 17,00 & 26,00 & & & \\
\hline & & Total & 290 & 20,98 & 2,15 & 17,00 & 29,00 & & & \\
\hline \multirow{9}{*}{$\begin{array}{l}\text { Knowledge } \\
\text { about cognition }\end{array}$} & \multirow{3}{*}{$\begin{array}{l}\text { Declarative Knowledge } \\
\text { (8-40 points) }\end{array}$} & Males & 87 & 27,41 & 5,78 & $14, \mathrm{OO}$ & 38,00 & \multirow{3}{*}{6681,5} & \multirow{3}{*}{$-3,289$} & \multirow{3}{*}{,OO 1 *** } \\
\hline & & Females & 203 & 24,66 & 6,99 & 10,00 & 40,00 & & & \\
\hline & & Total & 290 & 25,48 & 6,76 & 10,00 & 40,00 & & & \\
\hline & Procedural Knowledge & Males & 87 & 13,71 & 3,64 & 6,00 & 20,00 & & & \\
\hline & (4-20 points) & Females & 203 & 12,50 & 3,60 & 5,00 & 20,00 & 7131,0 & $-2,608$ & ,o09** \\
\hline & & Total & 290 & 12,86 & 3,65 & 5,00 & 20,00 & & & \\
\hline & & Males & 87 & 16,75 & 3,81 & 4,00 & 25,00 & & & \\
\hline & Conditional Knowledge & Females & 203 & 15,50 & 4,36 & 6,00 & 25,00 & 7332,0 & $-2,298$ &, $022^{*}$ \\
\hline & & Total & 290 & 15,87 & 4,24 & 4,00 & 25,00 & & & \\
\hline & & Males & 87 & 23,48 & 5,00 & 8,00 & 33,00 & & & \\
\hline & Planning & Females & 203 & 21,52 & 6,07 & 7,00 & 35,00 & 7087,0 & $-2,670$ & ,008** \\
\hline & & Total & 290 & 22,11 & 5,83 & 7,00 & 35,00 & & & \\
\hline & Management & Males & 87 & 31,72 & 5,35 & $15, \mathrm{OO}$ & $45, \mathrm{OO}$ & & & \\
\hline & Strategies & Females & 203 & 30,32 & 6,64 & $14, \mathrm{OO}$ & 63,00 & 7404,0 & $-2,185$ & ,029* \\
\hline & (9-45 points) & Total & 290 & 30,74 & 6,30 & $14, \mathrm{OO}$ & 63,00 & & & \\
\hline Regulation & & Males & 87 & 27,06 & 5,22 & $15, \mathrm{OO}$ & 38,00 & & & \\
\hline cognition & Comprehension Monitoring & Females & 203 & 25,33 & 6,23 & 11,00 & 40,00 & 7379,0 & $-2,222$ & ,026* \\
\hline & & Total & 290 & 25,85 & 5,99 & 11,00 & 40,00 & & & \\
\hline & & Males & 87 & 17,78 & 3,68 & 5,00 & 25,00 & & & \\
\hline & Debugging Strategies & Females & 203 & 17,20 & 3,63 & 8,00 & 25,00 & 7933,5 & $-1,377$ &, 169 \\
\hline & & Total & 290 & 17,38 & 3,65 & $5, \mathrm{OO}$ & 25,00 & & & \\
\hline & & Males & 87 & 20,55 & 3,77 & 12,00 & 30,00 & & & \\
\hline & Evaluation & Females & 203 & 19,20 & 4,46 & 9,00 & 30,00 & 7165,0 & $-2,554$ &, $011^{*}$ \\
\hline & & Total & 290 & 19,61 & 4,30 & 9,00 & 30,00 & & & \\
\hline
\end{tabular}


Table-5. Spearman rank order correlation coefficients among sport participation and metacognitive variables in Turkish male and female university students.

\begin{tabular}{l|c|c}
\hline & \multicolumn{2}{c}{ Sport participation } \\
\hline Metacognitive variables & Males & Females \\
\hline Knowledge about cognition & & \\
\hline Declarative knowledge &, 042 &,$- 249^{* *}$ \\
\hline Procedural knowledge &, 042 &,$- 194^{* *}$ \\
\hline Conditional knowledge &, 017 &,$- 230^{* *}$ \\
\hline Regulation of cognition & & \\
\hline Planning &, 002 &,$- 207^{* *}$ \\
\hline Information management &, 007 &,$- 173^{* *}$ \\
\hline Comprehension monitoring &, 006 &,$- 219^{* *}$ \\
\hline Debugging strategies &,- 011 &,$- 126^{* *}$ \\
\hline Evaluation &, 026 &,$- 146^{* *}$ \\
\hline *** & & \\
\hline
\end{tabular}

\section{Discussion}

Metacognitive awareness skill consisted of two components including knowledge about cognition and regulation of cognition. Knowledge about cognition includes the sub-dimensions of declarative knowledge, procedural knowledge and conditional knowledge. Regulation of cognition is related the sub-dimensions of planning, information management strategies, comprehension monitoring, debugging strategies and evaluation (Schraw and Moshman, 1995). Declarative knowledge refers to knowledge about the self and about the personal strategies, procedural knowledge indicates to knowledge about how to use these strategies, on the other hand, conditional knowledge implies to knowledge about when and why to use strategies. Regulation of cognition refers to activities that are aimed at regulating or controlling learning such as planning, information management strategies, comprehension monitoring, debugging strategies and evaluation of the learning process (Brown, 1987; Baker, 1989; Artz and Armour-Thomas, 1992).

It is well known that an athlete's planning, monitoring and evaluation of his or her learning processes can contribute greatly to them to develop their athletic performance. Many developing athletes can not reflect their own level of understanding of metacognitive strategies and cannot think of their skill levels correctly. Therefore, carefully expressed encouragements and planned interactions during practice can help athletes identify their own mistakes and at the same time raise awareness of their level of performance. The important thing to know is that metacognition is not only thinking, but also thinking about a person's own thinking process. Monitor and reflection of behavior to develop mature and successful athletes is a long-term task. The findings of an investigation indicate that metacognitive processes may be fundamental to effective cognitive control during the athletic development stages (Dail, 2014).

In a study aiming to compare the metacognitive skill levels between successful and unsuccessful teams in the Turkish Men's Second Volleyball League, no significant difference was found with respect to metacognitive skills between successful and unsuccessful teams' mean values except debugging strategies. The mean of debugging strategies in top three ranking team was $10.58 \%$ lower than unsuccessful teams. This means that top three rankings teams made fewer mistakes than unsuccessful teams experienced more errors with higher mean of debugging strategies. Execution volleyball skills without errors are critical factor for high performance (Sevimli, $2018)$.

The present study highlights the relationship between engaging in sports and cognitive functions in adolescents. So, this study compares the metacognitive awareness skill levels between athletes and sedentary Turkish male and female University Students. It has been reported that physical and sportive activities can improve children's health and cognitive and academic performance. On the other hand, excessive sedentary lifestyle has been shown to have negative effects on cognitive functions and academic achievement in children and adolescents (Syväoja, 2014).

Results of this study showed that there was no significant difference between sedentary and athlete participants in all sub-dimensions in male group. In female group, sedentary participants had significantly higher values than athlete counterparts in all sub-dimensions of MAS. Our study showed that cognitive awareness skills did not differ depending on participation in sports activities. Also, there were negative significant relationships between participation sport activities and all sub-dimensions of MAS scores in female while observing no significant relations among variables in males. The relationship between regular participation in sporting activities and cognitive functioning is well known (Ellemberg and St-Louis-Deschênes, 2010). The male and female athletes in our study were students from coaching and recreation departments of the Faculty of Sport Sciences, while the sedentary participants were students from the faculty of education and the faculty of economics and administrative sciences. Matriculation exam scores of sedentary participants are generally higher than athletes. Also their curriculum contains the topics related to metacognitive skills. This may be an advantage for sedentary participants. Since participation level in sport activities reported by athletes may be overestimated, there are insignificant differences between two athlete and sedentary groups in the physical fitness components. We have used selfreported measures of sportive activity and sedentary behavior in this study. Physical activity seems to have a potential that is not well understood and underused value to support learning. In addition, evidence of the positive effects of physical activity in healthy children and adolescents and the negative effects of sedentary behavior on cognitive functions and academic achievement are still based on inconsistent and limited research data. In generally, many studies reported conflicting results on the effect of sport on cognitive functions due to their limitations of designed study. Some of these studies report positive effects, while other studies do not confirm the positive effect of sportive activity on cognitive functions (Ahamed et al., 2007; Syväoja, 2014; Fels et al., 2015; Lambrick et al., 2016).

In sedentary group in our study, females had significantly higher values than males in the sub-dimensions of declarative knowledge, procedural knowledge, conditional knowledge and evaluation while males had significantly 
higher values than females in all sub-dimensions except debugging strategies in athlete group. Similar to our results, several studies showed inconsistent results regarding differences in metacognitive skills levels between male and female students. Some studies have reported that metacognitive skills of boys and girls differ, while other studies do not show significant differences (Niemivirta, 1997; Bidjerano, 2005; Virtanen and Nevgi, 2010). These findings demonstrate that both girls and boys use their metacognitive skills during learning (Niemivirta, 1997; Bidjerano, 2005; Virtanen and Nevgi, 2010).

The findings of the research conducted by Liliana and Lavinia (2011) demonstrate that both girls and boys use metacognitive skills during learning. In this study, as a result of the motivation, effort and matriculation exam scores of a student, there were significant differences between boys and girls in the sub-dimensions of MAS. Similarly, Liliana and Lavinia (2011) reported that there were significant differences between boys and girls solely on the following dimensions: the performance perception, perceptions of teachers about learning expectations, use of previous knowledge in problems, solving their own intellectual strengths and weaknesses, planning, accumulation of knowledge, using various learning strategies and monitoring of learning process. Such information may be useful in developing educational programs for young university students to develop their cognitive functions that are important to a particular sport discipline. Thus, it seems possible that these results of studies can be used in educational applications including teaching and learning in sport context.

It can be concluded that females in the sedentary group and male in the athlete group had significantly higher MAS values. Participation in sport activities had no positive effect on MAS scores in both genders due to selfreported measures of sportive activity. Superiority of sedentary females in MAS compare to athlete females may be attributed to their higher matriculation exam scores. In addition, it can be considered that there is no effective physical and sportive activity in females to make a difference in cognitive functions. Moreover, it can be estimated that the level of sports participation and quality of female athletes are very low.

Although physical activity has emerged as one of the most favorable methods for positively influencing cognitive function (Torres et al., 2018), the results of this study showed that male athletes had mathematically higher mean values in the metacognitive awareness skill levels than sedentary counterparts. Males also had higher means of MAS than females in both athletes and sedentary groups. Inefficacy of sportive activity on MAS level of female athletes may be partly due to their overrated physical activity level and lower matriculation exam scores compare to sedentary counterparts.

Further research is required to test the relationship between being athlete and metacognitive awareness skills in male and female university students. This study should be repeated on a well-designed larger scale and may confirm that raising metacognitive awareness levels between athlete sedentary students is desirable. Increasing metacognitive awareness due to participating sport activities may enhance the development of cognitive functions during their long term development stages.

\section{References}

Ahamed, Y., H. MacDonald, K. Reed, P.-J. Naylor, T. Liu-Ambrose and H. Mckay, 2007. School-based physical activity does not compromise children's academic performance. Medicine and Science in Sports and Exercise, 39(2): 371-376.Available at: https://doi.org/10.1249/01.mss.0000241654.45500.8e.

Akın, A., R. Abacı and B. Cetin, 2007. The validity and reliability study of the Turkish version of the metacognitive awareness inventory. Educational Science: Theory \& Practice, 7(2): 655- 680.

Artz, A.F. and E. Armour-Thomas, 1992. Development of a cognitive-metacognitive framework for protocol analysis of mathematical problem solving in small groups. Cognition and Instruction, 9(2): 137-175.Available at: https://doi.org/10.1207/s1532690xci0902_3.

Baker, L., 1989. Metacognition, comprehension monitoring, and the adult reader. Educational Psychology Review, 1(1): 3-38.Available at: https://doi.org/10.1007/bfo 1326548.

Bidjerano, T., 2005. Gender differences in self-regulated learning. Paper Presented at the 36th/2005 Annual Meeting of the Northeastern Educational Research Association, Kerhonkson, NY

Brown, A.L., 1987. Metacognition, executive control, self-regulation, and other more mysterious mechanisms. In F.E., Weinert, R.H., Kluwe (Ed.), Metacognition, Motivation and Understanding. Hillsdale, NJ: Lawrence Erlbaum.

Dail, T.K., 2014. Metacognition and coaching: How to develop a thinking athlete: Editor: Ferman konukman. Journal of Physical Education, Recreation and Dance, 85(5): 49-51.Available at: https://doi.org/10.1080/07303084.2014.897577.

Ellemberg, D. and M. St-Louis-Deschênes, 2010. The effect of acute physical exercise on cognitive function during development. Sport, Exercise, and Performance Psychology, 1 1(2): 122-126.Available at: https://doi.org/10.1016/j.psychsport.2009.09.006.

Erickson, K.I., C. Hillman, C.M. Stillman, R.M. Ballard, B. Bloodgood, D.E. Conroy, R. Macko, D.X. Marquez, S.J. Petruzzello and K.E, Powell, 2019. Physical activity, cognition, and brain outcomes: A review of the 2018 physical activity guidelines. Medicine and Science in Sports and Exercise, $51(6)$ : 1242-1251.Available at: https://doi.org/10.1249/mss.0000000000001936.

Fels, v.d.I.M., S.C. te Wierike, E. Hartman, M.T. Elferink-Gemser, J. Smith and C. Visscher, 2015. The relationship between motor skills and cognitive skills in 4-16 year old typically developing children: A systematic review. Journal of Science and Medicine in Sport, 18(6): 697-703.Available at: https://doi.org/10.1016/j.jsams.2014.09.007.

Flavell, J.H., 1979. Metacognition and cognitive monitoring: A new area of cognitive-developmental inquiry. American Psychologist, 34(10): 906-911.Available at: https://doi.org/10.1037//0003-066x.34.10.906.

Kubesch, S., 2002. Physical education: Training for body and mind. Neurology, 21(9): 487-490.

Lambrick, D., L. Stoner, R. Grigg and J. Faulkner, 2016. Effects of continuous and intermittent exercise on executive function in children aged 8-10 years. Psychophysiology, 53(9): 1335-1342.Available at: https://doi.org/10.1111/psyp.12688.

Liliana, C. and H. Lavinia, 2011. Gender differences in metacognitive skills. A study of the 8th grade pupils in Romania. Procedia-Social and Behavioral Sciences, 29: 396-401.Available at: https://doi.org/10.1016/j.sbspro.2011.11.255.

Lipowski, M., M. Lipowska, M. Jochimek and D. Krokosz, 2016. Resiliency as a factor protecting youths from risky behaviour: Moderating effects of gender and sport. European Journal of Sport Science, 16(2): 246-255.Available at: https://doi.org/10.1080/17461391.2015.1024755.

Mitchell, S.A., L.L. Griffin and J.L. Oslin, 1994. Tactical awareness as a developmentally appropriate focus for the teaching of games in elementary and secondary physical education. Physical Educator, 5 1(1): $21-28$.

Niemivirta, M., 1997. Gender differences in motivational-cognitive patterns of self-regulated learning. Paper Presented at the Annual Meeting of the American Educational Research Association, Chicago, IL.

Schraw, G. and R.S. Dennison, 1994. Assessing metacognitive awareness. Contemporary Educational Psychology, 19(4): 460-475.

Schraw, G. and D. Moshman, 1995. Metacognitive theories. Educational Psychology Review, 7(4): 351-371.

Sevimli, D., 2018. Comparison of the metacognitive awareness levels between successful and unsuccessful teams in the Turkish men's second volleyball league. Universal Journal of Educational Research, 6(12): 2715-2720.Available at: https://doi.org/10.13189/ujer.2018.061203. 
Syväoja, H., 2014. Physical activity and sedentary behaviour in association with academic performance and cognitive functions in schoolaged children. University of Jyväskylä Faculty of Social Sciences Department of Psychology, LIKES-Research Reports on Sport and Health 292, 20, 24 Finland.

Torres, A., B. Tennant, I. Ribeiro-Lucas, A. Vaux-Bjerke, K. Piercy and B. Bloodgood, 2018. Umbrella and systematic review methodology to support the 2018 physical activity guidelines advisory committee. Journal of Physical Activity and Health, 15(11): 805810.Available at: https://doi.org/10.1123/jpah.2018-0372.

Virtanen, P. and A. Nevgi, 2010. Disciplinary and gender differences among higher education students in self-regulated learning strategies. Educational Psychology, 30(3): 323-347.Available at: https://doi.org/10.1080/01443411003606391. 